\title{
Thermoelectric Performance of Tetrahedrite Synthesized by a Modified Polyol Process
}

\author{
Daniel P. Weller, ${ }^{\S}$ Daniel L. Stevens, ${ }^{\dagger}$ Grace E. Kunkel, $^{\dagger}$ Andrew M. Ochs, ${ }^{\dagger}$ Cameron F. Holder, ${ }^{\ddagger}$ \\ Donald T. Morelli, ${ }^{\S}$ and Mary E. Anderson*,† \\ ${ }^{\dagger}$ Chemistry Department, Hope College, Holland, Michigan 49423, United States \\ ${ }^{\S}$ Chemical Engineering and Materials Science Department, Michigan State University, East Lansing, Michigan 48824, United States \\ ${ }^{\ddagger}$ Chemistry Department, Pennsylvania State University, State College, Pennsylvania 16801, United States
}

\author{
Supporting Information
}

\begin{abstract}
Tetrahedrite, a promising thermoelectric material composed of earth-abundant elements, has been fabricated utilizing the rapid and low energy modified polyol process. Synthesis has been demonstrated for undoped and zinc-doped tetrahedrite samples on the gram scale requiring only $1 \mathrm{~h}$ at $220{ }^{\circ} \mathrm{C}$. This method is capable of incorporating dopants and producing particles in the 50-200 nm size regime. For determination of bulk thermoelectric properties, powders produced by this solution-phase method were densified into pellets by spark plasma sintering. Thermopower, electrical resistivity, and thermal conductivity were obtained for temperatures ranging from 323 to $723 \mathrm{~K}$. Maximum ZT

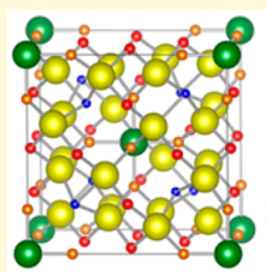

$\mathrm{Cu}^{2+}, \mathrm{Sb}^{3+}, \mathrm{S}$ polyol, $\mathrm{NaBH}_{4}$
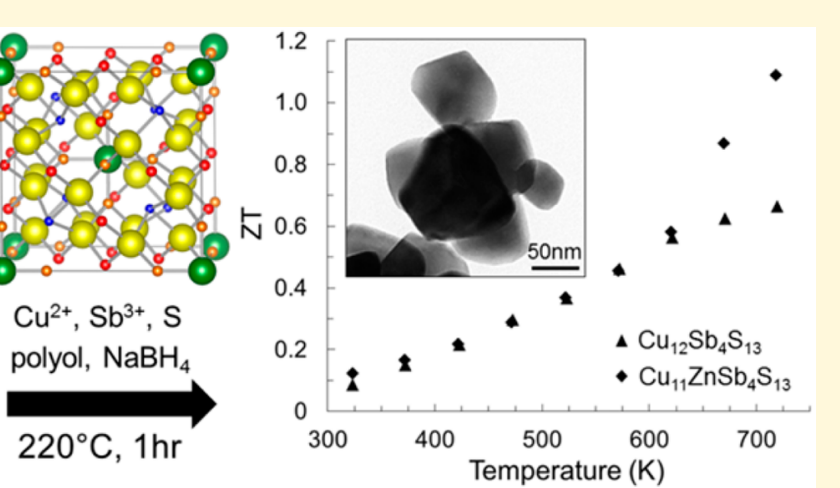
values at $723 \mathrm{~K}$ were found to be 0.66 and 1.09 for the undoped and zinc-doped tetrahedrite samples, respectively. These values are comparable to or greater than those obtained using time and energy intensive conventional solid-state methods. Consolidated pellets fabricated using nanomaterial produced by this solution-phase method were found to have decreased thermal conductivity, increased electrical resistivity, and increased thermopower. Exceptionally low total thermal conductivity values were found (below $0.7 \mathrm{~W} \mathrm{~m}^{-1} \mathrm{~K}^{-1}$ for undoped tetrahedrite and $0.5 \mathrm{~W} \mathrm{~m}^{-1} \mathrm{~K}^{-1}$ for zinc-doped tetrahedrite), with both having lattice thermal conductivities below $0.4 \mathrm{~W} \mathrm{~m}^{-1} \mathrm{~K}^{-1}$. This study explores how nanostructuring and doping of tetrahedrite via a solution-phase polyol process impacts thermoelectric performance.
\end{abstract}

\section{INTRODUCTION}

Within the current global energy infrastructure for electricity generation and transportation for industrial, commercial, and residential needs, it is estimated that only $28 \%$ of the energy consumed is utilized. ${ }^{1}$ Owing to the inefficient conversion of energy resources, most of the rejected energy goes to waste primarily in the form of latent heat released into the environment. Thermoelectric materials provide a means of capturing this waste heat for conversion into electricity via devices that require no moving parts or maintenance. The use of thermoelectrics for electrical power generation as well as for solid-state heating or cooling has been an active area of research since the 1960s, with significant advances obtained over the past decade improving the efficiency of these materials more than doublefold. ${ }^{2-5}$

The efficiency of a thermoelectric material operating at some temperature is described by the dimensionless figure of merit $(Z)$, where $Z T=\left(S^{2} \sigma / \kappa\right) T$ consisting of thermopower $(S)$, electrical conductivity $(\sigma)$, thermal conductivity $(\kappa)$, and absolute temperature $(T) .^{2-8}$ The figure of merit is maximized over a given temperature range, determining the type of application for which the material is appropriate. It has been found that $63 \%$ of rejected heat is at temperatures below 100 ${ }^{\circ} \mathrm{C}$ and $21 \%$ is at temperatures above $300{ }^{\circ} \mathrm{C}$ with the remaining in between the two temperatures. ${ }^{1}$ Bismuth telluride $\left(\mathrm{Bi}_{2} \mathrm{Te}_{3}\right)$ and lead telluride ( $\left.\mathrm{PbTe}\right)$ are the most highly studied thermoelectric materials with the former being useful for lower temperature applications and the latter finding application at higher temperatures.

For the variables comprising the figure of merit, a strong interdependence exists for the electrical and thermal properties. The Seebeck coefficient, or thermopower, describes the voltage generated by a temperature difference across the material. Electrical conductivity is directly proportional to the carrier concentration $(n)$ and mobility $(\mu)$ within the material according to $\sigma=n e \mu$, where $e$ is the elementary charge of an electron or hole. ${ }^{5}$ However, thermopower is inversely proportional to the carrier concentration. A power factor (PF), defined as $\mathrm{PF}=S^{2} \sigma$, is often presented to describe the potential of a material for power generation; and a large power factor

Received: November 21, 2016

Revised: February 4, 2017

Published: February 6, 2017 
indicates that a large voltage and high current will be produced. ${ }^{8}$ Since thermopower and electrical conductivity are both affected by the carrier concentration, chemical composition must be optimized to result in a maximum value for the product of $S^{2}$ and $\sigma .{ }^{6,7}$ Thermal conductivity describes the ability of a material to conduct heat, and it is composed of two components with the first describing the transport of heat via electrons and holes $\left(\kappa_{\mathrm{e}}\right)$ and the second contribution originating from phonons traveling through the crystal lattice $\left(\kappa_{\mathrm{L}}\right) .^{5}$ Like the inverse relationship of thermopower and electrical conductivity, an undesirable increase in thermal conductivity occurs as electrical conductivity increases, due to a rise in the electronic thermal conductivity. Accordingly, a balance must be struck between decreasing thermal conductivity while optimizing electrical conductivity.

To improve the figure of merit of prospective thermoelectric materials, researchers seek to maximize thermopower and electrical conductivity, while minimizing thermal conductivity. Both the thermopower and electrical conductivity are tailored to obtain a large power factor primarily by controlling the composition and concentration of carriers via dopants within narrow band gap semiconductor materials. ${ }^{6}$ To obtain low thermal conductivity, multiple approaches have been pursued to increase phonon scattering. Materials with complex unit cells possessing phonon glass electron crystal properties have successfully improved thermoelectric performance. ${ }^{3,5-7,9}$ In this approach, a crystal structure incorporating loosely bound "rattler" atoms will scatter phonons to further reduce the thermal conductivity. ${ }^{6-8}$ Alternatively, nanostructuring a material to produce a large number of grain boundary interfaces has been shown to decrease the thermal conductivity, as has the introduction of dopants within the unit cell to create point defects. ${ }^{3,5,7,8}$ An enhancement in the thermopower has also been observed for nanostructured powders because grain boundaries scatter electrons differently based on their wavelength resulting in electron energy filtering. ${ }^{10,11}$ Therefore, many strategies such as adding dopants, manipulating the crystal lattice, and controlling micro- and nanostructure have been shown to improve $Z T$.

One of the most prominent examples of nanostructuring to improve the thermoelectric performance involves bismuthantimony-telluride alloys where extensive ball milling created nanopowders with grain boundaries that were effective at scattering the phonons while only slightly increasing the electrical conductivity. For this material, a $Z T$ of 1.4 at $373 \mathrm{~K}$ was found. ${ }^{12}$ In another example, a $Z T$ of $\sim 2.2$ at $915 \mathrm{~K}$ has been achieved by fabricating mesostructured $\mathrm{PbTe}$ infiltrated with SrTe nanostructures through the use of powder processing and spark plasma sintering. ${ }^{13}$

While most thermoelectric materials contain low abundance elements or toxic metals (i.e., $\mathrm{Te}$ and $\mathrm{Pb}$, respectively), tetrahedrite is composed of light, earth-abundant, and nontoxic elements. The composition of tetrahedrite can range from copper-deficient to copper-rich varying between the $\mathrm{Cu}_{12} \mathrm{Sb}_{4} \mathrm{~S}_{13}$ and $\mathrm{Cu}_{14} \mathrm{Sb}_{4} \mathrm{~S}_{13}$ stoichiometries. ${ }^{14}$ In fact, this material exists as a common, naturally occurring sulfomineral with a range of different elements on the $\mathrm{Cu}$ (i.e., $\mathrm{Ag}, \mathrm{Zn}, \mathrm{Fe}, \mathrm{Pb}, \mathrm{Hg}, \mathrm{Ni}, \mathrm{Co}$ ) and $\mathrm{Sb}$ (i.e., $\mathrm{Te}, \mathrm{Bi}, \mathrm{As}$ ) sites. ${ }^{15,16}$ Tetrahedrite is a p-type semiconductor with current research investigating applications in photovoltaics with its potential as a solar absorber, as well as in thermoelectrics with its intrinsically low thermal conductivity attributed to its complex crystal structure. ${ }^{16,17}$ The crystal structure, shown in Figure 1, includes a large number of atoms

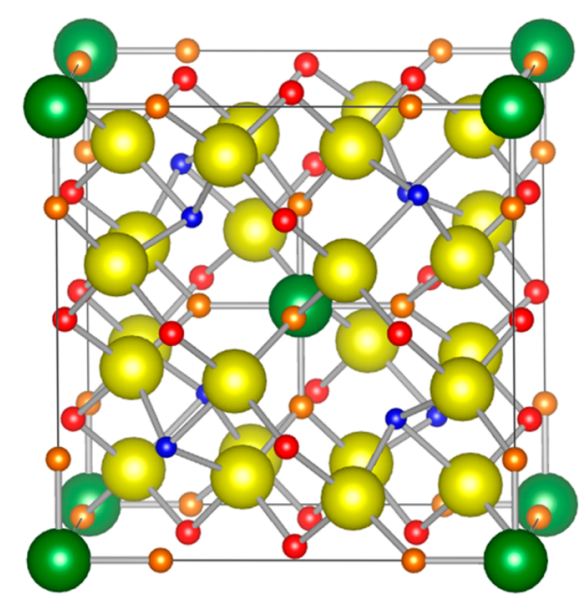

Figure 1. Tetrahedrite unit cell is cubic with $I \overline{4} 3 m$ symmetry. Copper atoms are shown in red $(\mathrm{Cu} 12 \mathrm{~d})$ and orange $(\mathrm{Cu} 12 \mathrm{e})$, sulfur atoms are shown in green (S2a) and yellow (S24g), and antimony atoms are shown in blue. Each $\mathrm{Cu} 12 \mathrm{~d}$ atom is tetrahedrally coordinated to four S24g atoms. Each Cu12e atom is trigonally coordinated to two S24g atoms and one $\mathrm{S} 2 \mathrm{a}$ atom, and $\mathrm{Sb}$ lone pairs are oriented toward the $\mathrm{Cu} 12 \mathrm{e}$ atom forming a trigonal bipyramid with the trigonal plane. ${ }^{14}$

(58) with a high symmetry unit cell (cubic, space group $\bar{I} 3 \mathrm{~m}) .{ }^{16}$ The chemical structure is composed of two copper positions (12d and $12 \mathrm{e})$, one antimony position, and two sulfur positions (S2a and S24g). ${ }^{15,16,18}$ The antimony lone pairs displace trigonally coordinated copper atoms, resulting in asymmetric bonding and an anharmonic rattling mode that suppresses acoustic phonons and contributes to phonon scattering. ${ }^{18}$

Tetrahedrite has been shown to be a good candidate for thermoelectric applications in the intermediate to high temperature range with a $Z T$ of $\sim 0.6$ at $673 \mathrm{~K}$ obtained for the undoped compound. ${ }^{19}$ The lattice thermal conductivity in these materials is found to be comparable to that of amorphous solids, which have a phonon mean free path on the scale of the interatomic spacing. ${ }^{18-21}$ Incorporating dopants has been shown to stabilize the tetrahedrite phase and has been an active area of research to optimize thermoelectric performance. ${ }^{19,21-27}$ By doping tetrahedrite with $\mathrm{Zn}, \mathrm{Ni}$, or $\mathrm{Mn}$, figures of merit near unity (at $723 \mathrm{~K}$ ) have been obtained, specifically by increasing thermopower and decreasing thermal conductivity. ${ }^{16,19,21,28}$ The dopants tune the Fermi energy, optimize the Seebeck coefficient, decrease electronic thermal conductivity, and create point-defects that scatter phonons to reduce thermal conductivity. ${ }^{19,21,22,27,29}$ Although these dopants are also known to decrease electrical conductivity, this change is typically not significant enough to offset the benefits of the improved thermopower and thermal conductivity. ${ }^{16,21}$

The vast majority of tetrahedrite studies have focused on materials fabricated by conventional "top-down" methods, requiring high temperatures and long annealing times. In general, the process begins with high purity elemental starting materials in evacuated and sealed ampules placed in a furnace at high temperatures (793-973 K) for long time periods (ranging from 12 to $40+\mathrm{h}$ ) before being further annealed at slightly lower temperatures $(723-793 \mathrm{~K})$ for even longer time periods ( $25 \mathrm{~h}$ to 3 weeks). ${ }^{16}$ It is noteworthy that metal sulfides are difficult to synthesize via these conventional means due to the volatility of sulfur. ${ }^{26}$ A "rapid" method was recently published, utilizing the energy-intensive means of mechanical alloying 
(ball-milling) for $8 \mathrm{~h}$ to obtain nickel-doped tetrahedrite, resulting in a $Z T$ of $\sim 0.75$ at $700 \mathrm{~K}^{26}$

A known advantage for "bottom up" methodologies is that they are ideal approaches for the production of nanostructured materials, which can in turn reduce the thermal conductivity of the thermoelectric materials. ${ }^{8}$ Techniques that have been successful at the production of thermoelectric materials, such as $\mathrm{Bi}_{2} \mathrm{Te}_{3}, \mathrm{PbTe}, \mathrm{BiSbTe}$, and $\mathrm{CoSb}_{3}$, are hydro- and solvothermal synthesis, ${ }^{30-38}$ hot-injection nanoparticle synthesis, ${ }^{39-51}$ coprecipitation, ${ }^{52-56}$ and microwave reactions. ${ }^{57-60}$ However, the scale of these synthetic methods is typically too low to permit bulk measurements. In the instances where enough material is produced for complete thermoelectric characterization, it is common that maximum $Z T$ s for the materials produced by solution-phase techniques are much lower than those produced by conventional methods.

Copper-antimony-sulfide compounds, including tetrahedrite, have been fabricated by solution-phase methods previously. ${ }^{30,34,39-42}$ The primary synthetic process is the hotinjection method, which is a typical technique used to produce nanoparticles. These particles have been investigated for their optical properties with the tunability of the band gap demonstrated based on composition and quantum size effects. ${ }^{39-41}$ This method was used to synthesize $\mathrm{Cu}_{3} \mathrm{SbSe}_{4}$ nanoparticles that were evaluated for their thermoelectric properties, finding a $Z T$ of 0.25 at $575 \mathrm{~K}$ with an increase to 0.5 obtained after doping the antimony site with tin. ${ }^{42}$

A solvothermal method has also successfully produced tetrahedrite powders, requiring that the sample be heated in a stainless steel bomb in a furnace at the relatively low temperature of $155{ }^{\circ} \mathrm{C}$ for $20-24 \mathrm{~h}^{30,34}$ Powders were produced with a yield of $250 \mathrm{mg}$ per batch, and the reported $Z T$ value was 0.63 at $720 \mathrm{~K}$, consistent with what had been produced via traditional solid-state methods. ${ }^{30}$ It was also found that the $Z T$ could be increased to 0.85 at $720 \mathrm{~K}$ by mixing naturally occurring tetrahedrite mineral with the sample.

Herein, a solution-phase, solid-state synthesis technique known as the modified polyol process was used to synthesize undoped and zinc-doped tetrahedrite. In this method, a high boiling point $\left(\sim 200\right.$ to $\left.300{ }^{\circ} \mathrm{C}\right)$ polyalcohol solvent is used to reduce metal ions, as the solvent can act as a reducing agent at elevated temperatures, and to induce diffusion of elemental components to form the desired product. The process here is modified by the addition of another reducing agent, such as $\mathrm{NaBH}_{4}$, and this synthetic method has been shown to synthesize thermoelectric materials with particles on the nanoscale. Previously, versions of the polyol method have been utilized to produce other thermoelectric materials, such as $\mathrm{Bi}_{2} \mathrm{Te}_{3}, \mathrm{PbTe}, \mathrm{Bi}_{1.5} \mathrm{Sb}_{0.5} \mathrm{Te}_{3}$, and $\mathrm{CoSb}_{3} .{ }^{61-70}$

The successful synthesis of tetrahedrite has here been demonstrated after heating the reaction mixture for $1 \mathrm{~h}$ at $220^{\circ} \mathrm{C}$. This is a fast and low energy method for the fabrication of nanostructured thermoelectric materials. Oftentimes, one of the challenges of solution-phase methods is that they are performed on the milligram scale and scaling up is not always a straightforward process. However, this process has been scaled up to produce $2+$ grams of material to obtain thermoelectric properties from a single batch synthesis (an uncommon feat for thermoelectric materials fabricated by solution-phase methods). While solution-phase approaches are useful for controlling and reducing particle size, the presence of capping agents or organic additives within powders produced using wet-chemistry methods will negatively affect the thermoelectric properties. ${ }^{6}$
It is noteworthy that the process described herein is a ligandand surfactant-free synthetic procedure producing particles in the $100 \mathrm{~nm}$ size regime. This study examines nanostructuring and doping of tetrahedrite via a solution-phase polyol process to investigate the impact on the thermoelectric properties. The synthetic product was characterized by powder X-ray diffraction and electron microscopy before undergoing processing to produce a pellet for interrogation of thermoelectric properties.

\section{EXPERIMENTAL SECTION}

Materials. All of the following reagents were used as received and purchased from Aldrich Chemical Co.: copper(II) acetate monohydrate $(\geq 98 \%)$, antimony(III) acetate $(\geq 99.99 \%)$, zinc(II) acetate (99.99\%), sulfur powder (99.98\%), and sodium borohydride (98\%). The solvents used were tetraethylene glycol (99\%) acquired from Alfa Aesar and anhydrous ethanol (200 proof, ACS/USP grade) obtained from Pharmco-Aaper.

Synthesis. For thermoelectric measurements on products collected from a single batch synthesis, tetrahedrite was synthesized on the two gram scale. Stoichiometric amounts of copper(II) acetate monohydrate $(3.99 \mathrm{~g}, 20.0 \mathrm{mmol})$, antimony(III) acetate $(1.97 \mathrm{~g}, 6.60$ $\mathrm{mmol})$, and sulfur powder $(0.694 \mathrm{~g}, 21.6 \mathrm{mmol})$ were combined with $200 \mathrm{~mL}$ of tetraethylene glycol in a $1 \mathrm{~L}$ round-bottom flask. The resulting blue-green solution was sparged with nitrogen gas for $10 \mathrm{~min}$ while it was continuously stirred. An excess amount of the reducing agent, sodium borohydride $(4 \mathrm{~g}, 100 \mathrm{mmol})$, was dissolved in $100 \mathrm{~mL}$ of tetraethylene glycol with the help of sonication. The resulting sodium borohydride solution was transferred slowly into the reaction flask, resulting in a black reaction mixture. The reaction was heated to $220{ }^{\circ} \mathrm{C}$ under positive nitrogen flow in a reflux apparatus, and held at this temperature for $1 \mathrm{~h}$. Then heat was removed so that the reaction flask could return to room temperature. The cooled reaction mixture was transferred into $50 \mathrm{~mL}$ centrifuge tubes and centrifuged at 5000 $\mathrm{rpm}$ for $10 \mathrm{~min}$. The resulting powder was then resuspended in ethanol, sonicated, and centrifuged again. This washing procedure was repeated three times and the product was consolidated. The black powder product was placed in a vacuum desiccator to dry.

For the synthesis of $\mathrm{Zn}$-doped tetrahedrite, stoichiometric amounts of copper(II) acetate monohydrate (3.66 g, $18.3 \mathrm{mmol})$, zinc(II) acetate $(0.306 \mathrm{~g}, 1.67 \mathrm{mmol})$, antimony(III) acetate $(1.97 \mathrm{~g}, 6.60$ $\mathrm{mmol})$, and sulfur powder $(0.694 \mathrm{~g}, 21.6 \mathrm{mmol})$ are utilized, and the procedure is the same as that described above. The resulting product was a dark, brick-red colored powder, indicating a change in the band gap of the material. This is consistent with the band structure calculations of $\mathrm{Lu}$ et al., which indicate that the band gap for $\mathrm{Zn}$-doped tetrahedrite narrows to approximately $0.8 \mathrm{eV}$ in $\mathrm{Cu}_{10} \mathrm{Zn}_{2} \mathrm{Sb}_{4} \mathrm{~S}_{13}$ from a value of approximately $1.2 \mathrm{eV}$ in the undoped material. ${ }^{19}$

Structural and Compositional Analysis. X-ray powder diffraction patterns were collected using a Rigaku Miniflex benchtop diffractometer with $\mathrm{Cu} \mathrm{K} \alpha$ radiation at $30 \mathrm{kV}$ and $15 \mathrm{~mA}$ over a $2 \theta$ range of $10-65^{\circ}$. Transmission electron microscopy (TEM) characterization was performed using a Tecnai G2 20 XTWIN operating at 80 $\mathrm{kV}$. Samples were prepared by drop-casting a dilute solution of nanoparticles from ethanolic solution onto a nickel TEM grid (Formvar/carbon 400 mesh). High resolution scanning electron microscopy (SEM) was done using a JEOL JSM-7200F-LV field emission microscope operating at $2 \mathrm{kV}$ with images obtained via the in-lens detector. Samples for SEM were prepared by dispersing the dry powder product on carbon tabs or carbon paint adhered to an SEM stub. Energy dispersive X-ray spectroscopy (EDS) data for these samples were collected using a Hitachi TM-3000 Tabletop Microscope with a Bruker XFlash MIN SVE detector and scan generator for EDS capability. The accelerating voltage of the electron beam was $15 \mathrm{kV}$. Data for multiple regions of the tetrahedrite samples were collected.

Processing. Resultant powders were loaded into a $10 \mathrm{~mm}$ graphite die and densified using a pulsed electric current sintering method in a Calnano 211-LX Dr. Sinter Spark Plasma Sintering (SPS) machine. Sintering was done at $300{ }^{\circ} \mathrm{C}$ for 10 min under $40 \mathrm{MPa}$ pressure in an argon atmosphere. To improve pellet density (from the $\sim 85 \%$ 
achieved), the pellets were then ball milled for $5 \mathrm{~min}$ and sintered at $350{ }^{\circ} \mathrm{C}$ for another $10 \mathrm{~min}$ under $40 \mathrm{MPa}$ pressure in argon. Room temperature density $(\rho)$ was measured using Archimedes method, and $92 \%$ density was achieved for both samples. Each pellet was then cut into a disc of about $1 \mathrm{~mm}$ thickness and rectangular slabs of about 3 $\mathrm{mm} \times 3 \mathrm{~mm} \times 7 \mathrm{~mm}$ using a diamond saw apparatus. Discs and rectangular slabs were polished using fine grit sandpaper. Crystal structure was maintained throughout this process as confirmed by powder X-ray diffraction using a Rigaku Miniflex-II benchtop diffractometer with $\mathrm{Cu} \mathrm{K} \alpha$ radiation over a $2 \theta$ range of $20^{\circ}$ to $90^{\circ}$.

Transport Property Measurements. Electrical properties (electrical resistivity and Seebeck coefficient) of the rectangular slabs were measured from 323-723 $\mathrm{K}$ using an ULVAC ZEM-3 machine. Discs with $1 \mathrm{~mm}$ thickness were used for thermal diffusivity (D) measurements in a Netzsch LFA-457 instrument from 323-723 K. By assuming the Dulong-Petit heat capacity $\left(C_{\mathrm{p}}\right)$, thermal conductivity was calculated according to $\kappa=\rho \mathrm{DC}_{\mathrm{p}}$. The sample is expected to be stable over the selected temperature range as tetrahedrite has been shown to be stable up to temperatures exceeding $725 \mathrm{~K}^{71}$ Uncertainty in thermopower, electrical resistivity, and thermal conductivity is assumed to be about $5 \%, 5 \%$, and $10 \%$, respectively. Consequently, an uncertainty of about $12 \%$ is realized for $Z T$.

\section{RESULTS AND DISCUSSION}

Structural and Compositional Analysis. Powder X-ray diffraction patterns for the postsynthetic products of both samples are displayed in Figure 2, along with a reference

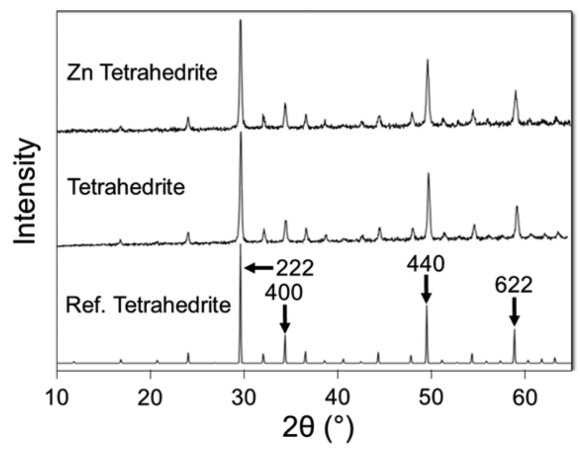

Figure 2. Powder XRD patterns for postsynthetic products of undoped and zinc-doped tetrahedrite. A reference XRD pattern for tetrahedrite is included, and the most four most intense peaks are indexed. ${ }^{14}$ Both samples match the reference with no secondary phases present.

pattern for pure phase tetrahedrite. ${ }^{15}$ Pure single-phase tetrahedrite was observed for both samples before and after powder processing. It is noteworthy that many past investigations have studied undoped tetrahedrite containing famatinite $\left(\mathrm{Cu}_{3} \mathrm{SbS}_{4}\right)$ as a secondary impurity phase. ${ }^{19,22,23,25,28,30,72,73}$ In addition, it has been shown that incorporating dopants into the tetrahedrite crystal structure is an effective method for stabilizing the pure phase of tetrahedrite. $^{22}$ The synthetic method described in this paper is advantageous in that single-phase tetrahedrite can be obtained in a relatively short amount of time without the inclusion of dopants. Additionally, this synthetic method has demonstrated successful production of the single-phase quaternary compound, zinc-doped tetrahedrite.

SEM and TEM images of the postsynthesis undoped tetrahedrite sample are presented in Figure 3. Using this ligand and the surfactant-free synthetic method, both imaging techniques show particles in the range of 50-200 $\mathrm{nm}$ for the undoped and zinc-doped samples (see Supporting Informa-
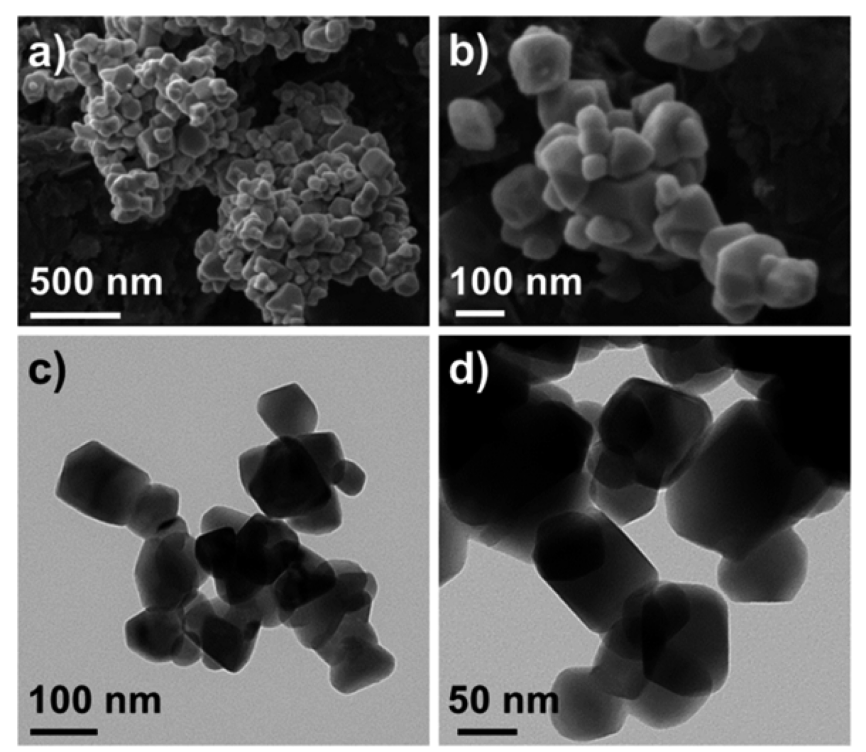

Figure 3. Representative $\operatorname{FESEM}(a, b)$ and TEM (c,d) images of undoped tetrahedrite. These show a distribution of particle sizes ranging from approximately $50-200 \mathrm{~nm}$.

tion). The size and shape of the particles are maintained after powder processing as observed by SEM imaging of the pellet (see Supporting Information). While these sizes are outside the quantum regime, nanostructuring on this scale has been shown to successfully increase the thermopower and decrease the thermal conductivity. ${ }^{7,8,10}$ A key variable studied herein, enabled by this synthetic method, is how this particle size will affect the thermoelectric properties of tetrahedrite and zincdoped tetrahedrite. A previous study done by $\mathrm{Lu}$ et al. investigated a solid solution of zinc-doped tetrahedrite $\left(\mathrm{Cu}_{12-x} \mathrm{Zn}_{x} \mathrm{Sb}_{4} \mathrm{~S}_{13}\right.$ for $x=0$ to 1.5$)$, and the $x=1$ sample had a higher $Z T$ than the other compositions for comparable temperatures. ${ }^{19}$ Thus, $\mathrm{Cu}_{11} \mathrm{ZnSb}_{4} \mathrm{~S}_{13}$ was chosen as the target composition for the zinc-doped sample in this study.

EDS data obtained for both the doped and undoped tetrahedrite indicate a copper-enriched composition was obtained. When the stoichiometric ratios are normalized relative to the sulfur, the formula obtained for the undoped tetrahedrite was $\mathrm{Cu}_{13.6 \pm 0.1} \mathrm{Sb}_{4.3 \pm 0.2} \mathrm{~S}_{13.0 \pm 0.3}$ and for the zincdoped tetrahedrite was $\mathrm{Cu}_{12.6 \pm 0.2} \mathrm{Zn}_{1.2 \pm 0.1} \mathrm{Sb}_{4.2 \pm 0.1} \mathrm{~S}_{13.0 \pm 0.2}$. This copper-enrichment is consistent with the range of solid solution compositions of tetrahedrite being $\mathrm{Cu}_{12+x} \mathrm{Sb}_{4+y} \mathrm{~S}_{13}$, where 0.11 $\leq x \leq 1.77$ and $0.03 \leq y \leq 0.30 .{ }^{14}$ An $11: 1$ atomic ratio between copper and zinc in this sample is supported, within measurement error, by the EDS data. Elemental analysis after powder processing revealed that the stoichiometries were maintained (see Supporting Information).

Furthermore, reaction temperature and time were found to have an influence on the purity of the final product. To obtain pure phase tetrahedrite, the optimized reaction temperature and time were $220^{\circ} \mathrm{C}$ for $1 \mathrm{~h}$. At temperatures below $200{ }^{\circ} \mathrm{C}$, copper sulfide phases are first observed and then famatanite is obtained as the antimony is reduced and incorporated. This was also found to be the case for reactions carried out at $220{ }^{\circ} \mathrm{C}$ for $45 \mathrm{~min}$ or less. With a reaction temperature of $240{ }^{\circ} \mathrm{C}$, multiple impurities were detected including elemental antimony, chalcostibite $\left(\mathrm{CuSbS}_{2}\right)$, and copper sulfide phases.

Thermoelectric Measurements. The thermopower, electrical resistivity, and power factor data are shown in Figure 4. As 

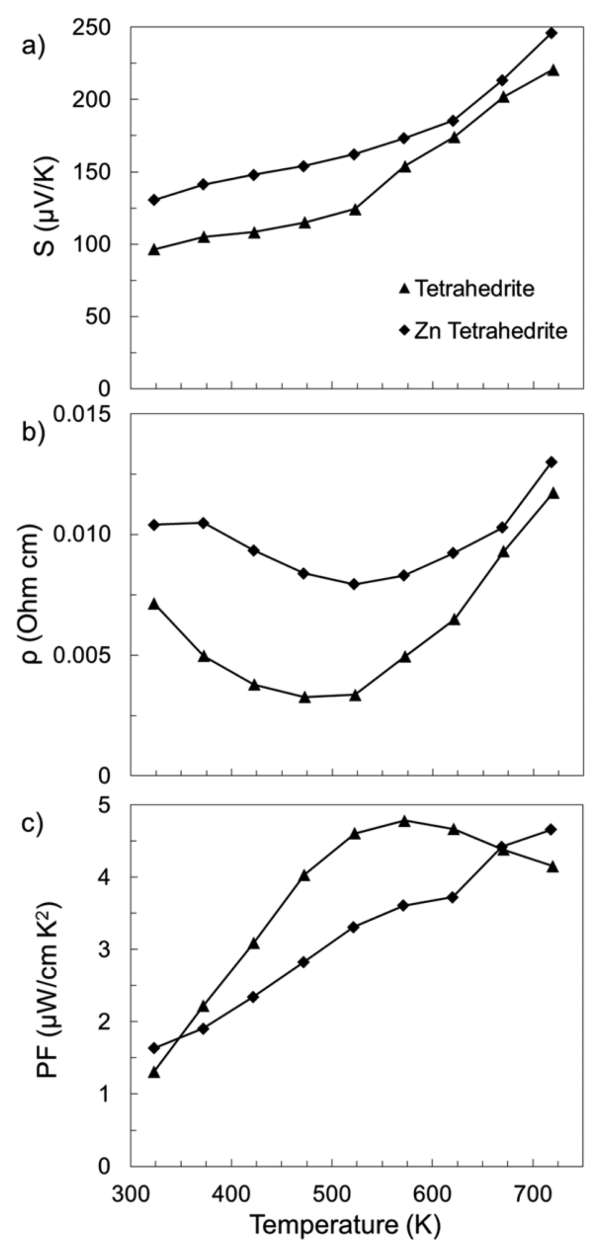

Figure 4. Electrical transport properties for undoped tetrahedrite (triangles) and zinc-doped tetrahedrite (diamonds) as a function of temperature from 323 to $723 \mathrm{~K}$. (a) The thermopower of the zincdoped sample is greater than the undoped tetrahedrite sample. Both samples achieve values exceeding $200 \mu \mathrm{VK}^{-1}$. (b) The zinc-doped sample is higher in electrical resistivity than the undoped tetrahedrite sample, and this is likely a result of the Fermi energy shifting due to the dopant. (c) Power factors converge to similar values at high temperature for both samples.

expected for a p-type semiconductor, the thermopower of both samples is positive. Additionally, a rise in thermopower is observed in both samples as temperature increases. This behavior is expected for a degenerate semiconductor, for which the thermopower is proportional to temperature. ${ }^{7}$ Differences in Seebeck coefficients between the two samples may be explained by considering the electronic band structure of tetrahedrite, in which the Fermi energy is located near the valence band edge. ${ }^{19}$ The zinc-doped sample demonstrates a higher thermopower than the undoped sample, which is likely a result of the zinc dopant shifting the Fermi energy into the band gap. ${ }^{19}$ The divalent zinc dopant effectively adds more electrons to the lattice (or fills more holes) and pushes the Fermi level to a higher energy. In this case, a lower carrier concentration will be correlated to an increase in thermopower, as observed for the zinc-doped sample. At the highest measured temperature $(723 \mathrm{~K})$, both samples exhibit thermopower values exceeding $200 \mu \mathrm{VK}^{-1}$, which is in some cases more than two times higher than those reported previously. ${ }^{19,21,30}$ These high Seebeck coefficients may be a consequence of the alteration of the electronic band structure due to nanostructuring effects. ${ }^{5,74}$
Alternatively, these higher values could result from an energy filtering process, where charge carriers are selectively scattered more efficiently according to their energies. ${ }^{10,11}$ By nanostructuring the samples in this investigation, grain boundary scattering has a significant effect and this increased scattering of charge carriers leads to an increase in the thermopower.

Electrical resistivity is plotted in Figure $4 b$, showing a lower resistivity in the undoped tetrahedrite sample versus the zincdoped sample. Both samples demonstrate a higher electrical resistivity than those fabricated by conventional bulk techniques, such as the ones reported by $\mathrm{Lu}$ et al., ${ }^{19}$ and rise somewhat faster above the minimum. This is not unexpected for these nanostructured materials because the increased grain boundary scattering of charge carriers will reduce efficient conduction of carriers and can also change the temperature dependence of the charge carrier mobility. Additionally, this behavior could be partly explained by the greater porosity in the samples, which were found to be about $92 \%$ dense compared to most samples which are often measured at $95 \%$ density or greater. However, the slightly greater porosity is only expected to have a minor impact on the samples' electrical properties.

The power factor, shown in Figure $4 c$, reaches about $4.5 \mu \mathrm{W}$ $\mathrm{cm}^{-1} \mathrm{~K}^{-2}$, which is on par with samples fabricated by conventional high temperature methods. ${ }^{19,21}$ Consistent with previous findings that investigated the effect of dopants in tetrahedrite, the power factor for the zinc-doped sample is lower than that of the undoped tetrahedrite over most of the temperature range because of its greater electrical resistivity. ${ }^{21}$ While an increase in electrical resistivity was observed for both samples, it was offset by the increased thermopower. Therefore, the samples synthesized in this experiment are equally useful for power generation at intermediate temperature ranges when compared with previously reported samples.

For a nanostructured thermoelectric material, more grain boundaries will be present in the sample and phonon scattering at these interfaces is expected to have a greater effect on the thermal conductivity. Thermal conductivity values for both samples are plotted in Figure 5. The overall shape of these curves is consistent with a combination of phonon-interface scattering (more dominant at low to intermediate temperatures) and phonon-phonon scattering (above the maximum). For the undoped tetrahedrite sample, these values are significantly lower than those ranging from 0.7 to $1.5 \mathrm{~W} \mathrm{~m}^{-1}$ $\mathrm{K}^{-1}$ obtained by other studies. ${ }^{19,30}$ Variation between the two samples tested in this study can, in part, be accounted for by mass-difference impurity scattering, occurring from increased point defects in the zinc-doped sample. This result agrees with previously reported behavior where the introduction of dopants caused a decrease in the thermal conductivity of tetrahedrite. ${ }^{19,21}$

As aforementioned, the thermal conductivity is the sum of two major components, the electronic thermal conductivity $\left(\kappa_{\mathrm{e}}\right)$ and the lattice thermal conductivity $\left(\kappa_{\mathrm{L}}\right)$. The electronic contribution to thermal conductivity is determined by the Wiedemann-Franz law, which states $\kappa_{\mathrm{e}}=L_{0} \sigma T$. In this expression, $L_{0}$ is the Lorenz number $\left(2.44 \times 10^{-8} \mathrm{~W} \Omega \mathrm{K}^{-2}\right.$ for free electrons in a metal), $\sigma$ is the electrical conductivity, and $T$ is temperature. The lattice thermal conductivity is calculated by $\kappa_{\mathrm{L}}={ }^{1} /{ }_{3} v_{\mathrm{s}} C_{\mathrm{v}} L_{\mathrm{ph}}$ where $v_{\mathrm{s}}$ is the velocity of sound, $C_{\mathrm{v}}$ is the heat capacity at constant volume, and $L_{\mathrm{ph}}$ is the mean free path length of phonons through the material. ${ }^{6,7}$ The electronic contribution was calculated by the Wiedemann-Franz law and 

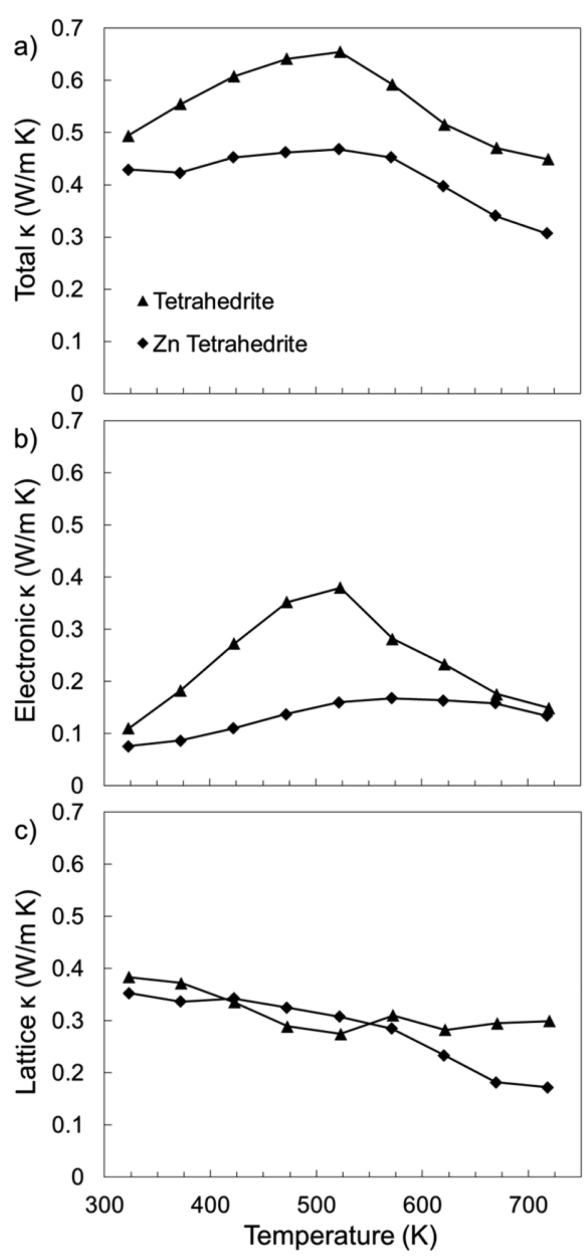

Figure 5. Thermal conductivity values for undoped tetrahedrite (triangles) and zinc-doped tetrahedrite (diamonds) as a function of temperature from 323 to $723 \mathrm{~K}$. (a) The total thermal conductivity values are low for both samples compared to most materials. The undoped tetrahedrite sample demonstrates a higher thermal conductivity than the zinc-doped sample. (b) The electronic thermal conductivity is larger in the undoped tetrahedrite sample than the zincdoped sample. (c) Lattice thermal conductivity is obtained by subtracting the electronic contribution from the total thermal conductivity. Both samples demonstrate exceptionally low lattice thermal conductivities, which are likely due to increased phonon scattering from grain boundaries, a complex unit cell, and point defect scattering in the zinc-doped sample.

subtracted from the total measured thermal conductivity to determine the lattice contribution.

Data for the electronic thermal conductivity and the lattice thermal conductivity are shown in Figure 5 panels $b$ and $c$, respectively. Because of its greater electrical conductivity, the tetrahedrite sample has a larger electronic thermal conductivity than the zinc-doped sample. After removing the electronic contribution from the total thermal conductivity, the lattice thermal conductivities of the two samples converge to similar values below $0.4 \mathrm{~W} \mathrm{~m}^{-1} \mathrm{~K}^{-1}$. Overall, it is notable that both the magnitude of the lattice thermal conductivity and its temperature dependence are lower than those reported for bulk samples at these compositions over the reported temperature range. ${ }^{19}$ This is consistent with additional phonon scattering from grain boundaries and a resulting reduction in phonon mean free path from nanostructuring. Thus, an exceptionally low lattice thermal conductivity was obtained due to a combination of phonon scattering from point defects, the complex crystal structure of tetrahedrite, and interfacial scattering occurring at grain boundaries.

As shown in Figure 6, peak ZT values at $723 \mathrm{~K}$ were found to be 0.66 and 1.09 for the undoped and zinc-doped samples,

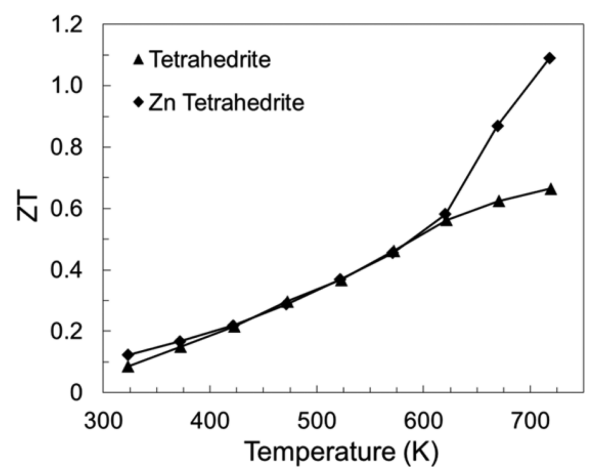

Figure 6. ZT values for undoped tetrahedrite (triangles) and zincdoped tetrahedrite (diamonds) as a function of temperature from 323 to $723 \mathrm{~K}$. The dimensionless figure of merit rises with increasing temperature for both samples. Values peak at $723 \mathrm{~K}$ with $Z T=0.66$ for the undoped tetrahedrite sample and $Z T=1.09$ for the zinc-doped sample.

respectively. The zinc-doped sample in this study exhibits a higher ZT than any other zinc-doped sample reported before. Previously, a maximum value of $Z T=0.70$ at $673 \mathrm{~K}$ was demonstrated for $\mathrm{Cu}_{11} \mathrm{ZnSb}_{4} \mathrm{~S}_{13}$ and $Z T=0.95$ at $723 \mathrm{~K}$ for $\mathrm{Cu}_{11.5} \mathrm{Zn}_{0.5} \mathrm{Sb}_{4} \mathrm{~S}_{13}$ by $\mathrm{Lu}$ et al. ${ }^{19}$ The effect of the nanostructuring on the undoped tetrahedrite sample increased the thermopower and decreased the thermal conductivity. Yet, these enhancements did not result in an increased $Z T$ relative to the bulk material because of the greater electrical resistivity. The ZT obtained for the undoped tetrahedrite sample is on par with the 0.6 value at $673 \mathrm{~K}$ obtained for materials made via solution or conventional techniques. ${ }^{16,19,21,30}$ Using this $1 \mathrm{~h}$ solution-phase process to generate tetrahedrite nanomaterial, a high figure of merit is obtained that is comparable to or greater than samples made by conventional methods.

\section{CONCLUSION}

This solution-phase method for the production of tetrahedrite yields a phase-pure, nanostructured product on the gram-scale via a fast and low energy process that is capable of incorporating desired dopants. The synthesis of this material is quite energy efficient compared to conventional fabrication, as this solution-phase process only requires $1 \mathrm{~h}$ at $220^{\circ} \mathrm{C}$. It has here been demonstrated that this wet chemistry method can be scaled up to synthesize pure crystalline product on the gram scale $(2+$ grams $)$. Phase-pure tetrahedrite is obtained by this process with particles in the 50-200 $\mathrm{nm}$ range. The incorporation of a zinc dopant into tetrahedrite was successful to synthesize pure single-phase material without the production of side products, yielding a quaternary compound.

This synthesis enabled the investigation of nanostructuring and doping in tetrahedrite to determine the effect on thermoelectric properties. Nanostructuring the undoped tetrahedrite did not increase the $Z T$ relative to the bulk material, but it did increase the thermopower double-fold and decrease the thermal conductivity two-fold. While the thermopower and thermal conductivity were improved, these enhance- 
ments were offset by the increase in the electrical resistivity. The overall performance of the undoped tetrahedrite is on par with that of material fabricated by more time and energy intensive conventional means. For the zinc-doped tetrahedrite sample synthesized via the solution-phase, the $Z T$ was increased relative to the undoped tetrahedrite made by the same method, as well as in comparison to other zinc-doped derivatives of tetrahedrite fabricated by conventional processing. While both the undoped and zinc-doped samples had a similar detrimental increase in electrical resistivity, the increase of the thermopower and the decrease of the thermal conductivity for the zinc-doped material were significant enough to offset the electrical resistivity increase. For the zinc-doped tetrahedrite, the resulting ZT of 1.09 at $723 \mathrm{~K}$ is, to the authors' knowledge, among the highest figures of merit reported for any thermoelectric material synthesized by solution-phase methodologies.

\section{ASSOCIATED CONTENT}

\section{S Supporting Information}

The Supporting Information is available free of charge on the ACS Publications website at DOI: 10.1021/acs.chemmater.6b04950.

Transmission electron microscopy characterization regarding the as-synthesized zinc-doped tetrahedrite sample as well as SEM and EDS data for both samples after powder processing (PDF)

\section{AUTHOR INFORMATION}

\section{Corresponding Author}

*E-mail: meanderson@hope.edu.

ORCID

Mary E. Anderson: 0000-0001-8369-2177

Notes

The authors declare no competing financial interest.

\section{ACKNOWLEDGMENTS}

This work was supported by NSF-CHE-1508244, NSF-CBET1507789, and the U.S. Dept. of Education GAANN program. Additional funding was provided by Hope College, the Towsley Foundation, and a generous gift to Hope College from the Herbert H. and Grace A. Dow Foundation. For technical assistance, we thank Prof. Ben Kopek at Hope College, Dr. Jennifer Misuraca from JEOL USA Inc., and Pennsylvania State University's MRI MCL. Visualization for Electronic and Structural Analysis (VESTA) was used for crystallographic modeling of the tetrahedrite unit cell.

\section{REFERENCES}

(1) Forman, C.; Muritala, I. K.; Pardemann, R.; Meyer, B. Estimating the Global Waste Heat Potential. Renewable Sustainable Energy Rev. 2016, 57, 1568-1579.

(2) Bell, L. E. Cooling, Heating, Generating Power, and Recovering Waste Heat with Thermoelectric Systems. Science 2008, 321, 14571461.

(3) Tan, G.; Zhao, L.-D.; Kanatzidis, M. G. Rationally Designing High-Performance Bulk Thermoelectric Materials. Chem. Rev. 2016, 116, 12123-12149.

(4) Goldsmid, H. J. Principles of Thermoelectric Devices. Br. J. Appl. Phys. 1960, 11, 209-217.

(5) Heremans, J. P.; Dresselhaus, M. S.; Bell, L. E.; Morelli, D. T. When Thermoelectrics Reached the Nanoscale. Nat. Nanotechnol. 2013, 8, 471-473.
(6) Tritt, T. M. Thermoelectric Phenomena, Materials, and Applications. Annu. Rev. Mater. Res. 2011, 41, 433-448.

(7) Snyder, G. J.; Toberer, E. S. Complex Thermoelectric Materials. Nat. Mater. 2008, 7, 105-114.

(8) Kanatzidis, M. G. Nanostructured Thermoelectrics: The New Paradigm? Chem. Mater. 2010, 22, 648-659.

(9) Slack, G. A. New Materials and Performance Limits for Thermoelectric Cooling. In CRC Handbook of Thermoelectrics; Rowe, D.., Ed.; CRC Press, 1995.

(10) Heremans, J. P.; Thrush, C. M.; Morelli, D. T. Thermopower Enhancement in Lead Telluride Nanostructures. Phys. Rev. B: Condens. Matter Mater. Phys. 2004, 70, 115334.

(11) Zide, J. M. O.; Vashaee, D.; Bian, Z. X.; Zeng, G.; Bowers, J. E.; Shakouri, A.; Gossard, A. C. Demonstration of Electron Filtering to Increase the Seebeck Coefficient in In0.53Ga0.47As/In0.53Ga0.28A10.19As Superlattices. Phys. Rev. B: Condens. Matter Mater. Phys. 2006, 74, 205335.

(12) Poudel, B.; Hao, Q.; Ma, Y.; Lan, Y.; Minnich, A.; Yu, B.; Yan, X.; Wang, D.; Muto, A.; Vashaee, D.; Chen, X.; Liu, J.; Dresselhaus, M. S.; Chen, G.; Ren, Z. High-Thermoelectric Performance of Nanostructured Bismuth Antimony Telluride Bulk Alloys. Science 2008, 320, 634-638.

(13) Biswas, K.; He, J.; Blum, I. D.; Wu, C.-I.; Hogan, T. P.; Seidman, D. N.; Dravid, V. P.; Kanatzidis, M. G. High-Performance Bulk Thermoelectrics with All-Scale Hierarchical Architectures. Nature 2012, 489, 414-418.

(14) Tatsuka, K.; Morimoto, N. Composition Variation and Polymorphism of Tetrahedrite in the Cu-Sb-S System below 400C. Am. Mineral. 1973, 58, 425-434.

(15) Wuensch, B. J. The Crystal Structure of Tetrahedrite, Cu12Sb4S13. Zeitschrift fur Krist. - New Cryst. Struct. 1964, 119, 437-453.

(16) Chetty, R.; Bali, A.; Mallik, R. C. Tetrahedrites as Thermoelectric Materials: An Overview. J. Mater. Chem. C 2015, 3, 1236412378.

(17) Heo, J.; Ravichandran, R.; Reidy, C. F.; Tate, J.; Wager, J. F.; Keszler, D. A. Design Meets Nature: Tetrahedrite Solar Absorbers. Adv. Energy Mater. 2015, 5, 1-7.

(18) Lai, W.; Wang, Y.; Morelli, D. T.; Lu, X. From Bonding Asymmetry to Anharmonic Rattling in Cu12Sb4S13 Tetrahedrites: When Lone-Pair Electrons Are Not so Lonely. Adv. Funct. Mater. 2015, 25, 3648-3657.

(19) Lu, X.; Morelli, D. T.; Xia, Y.; Zhou, F.; Ozolins, V.; Chi, H.; Zhou, X.; Uher, C. High Performance Thermoelectricity in EarthAbundant Compounds Based on Natural Mineral Tetrahedrites. Adv. Energy Mater. 2013, 3, 342-348.

(20) Lu, X.; Morelli, D. T.; Xia, Y.; Ozolins, V. Increasing the Thermoelectric Figure of Merit of Tetrahedrites by Co-Doping with Nickel and Zinc. Chem. Mater. 2015, 27, 408-413.

(21) Heo, J.; Laurita, G.; Muir, S.; Subramanian, M. A.; Keszler, D. A. Enhanced Thermoelectric Performance of Synthetic Tetrahedrites. Chem. Mater. 2014, 26, 2047-2051.

(22) Barbier, T.; Lemoine, P.; Gascoin, S.; Lebedev, O. I.; Kaltzoglou, A.; Vaqueiro, P.; Powell, A. V.; Smith, R. I.; Guilmeau, E. Structural Stability of the Synthetic Thermoelectric Ternary and NickelSubstituted Tetrahedrite Phases. J. Alloys Compd. 2015, 634, 253-262.

(23) Chetty, R.; Prem Kumar, D. S.; Rogl, G.; Rogl, P.; Bauer, E.; Michor, H.; Suwas, S.; Puchegger, S.; Giester, G.; Mallik, R. C. Thermoelectric Properties of a Mn Substituted Synthetic Tetrahedrite. Phys. Chem. Chem. Phys. 2015, 17, 1716-1727.

(24) Prem Kumar, D. S.; Chetty, R.; Rogl, P.; Rogl, G.; Bauer, E.; Malar, P.; Mallik, R. Thermoelectric Properties of Cd Doped Tetrahedrite: Cu12-xCdxSb4S13. Intermetallics 2016, 78, 21-29.

(25) Prem Kumar, D. S.; Chetty, R.; Femi, O. E.; Chattopadhyay, K.; Malar, P.; Mallik, R. C. Thermoelectric Properties of Bi Doped Tetrahedrite. J. Electron. Mater. 2016, 1-7.

(26) Barbier, T.; Rollin-Martinet, S.; Lemoine, P.; Gascoin, F.; Kaltzoglou, A.; Vaqueiro, P.; Powell, A. V.; Guilmeau, E. Thermoelectric Materials: A New Rapid Synthesis Process for Nontoxic and 
High-Performance Tetrahedrite Compounds. J. Am. Ceram. Soc. 2016, 99, 51-56.

(27) Lu, X.; Morelli, D. T.; Wang, Y.; Lai, W.; Xia, Y.; Ozolins, V. Phase Stability, Crystal Structure, and Thermoelectric Properties of Cu12Sb4S13-xSex Solid Solutions. Chem. Mater. 2016, 28, 17811786.

(28) Chetty, R.; Bali, A.; Naik, M. H.; Rogl, G.; Rogl, P.; Jain, M.; Suwas, S.; Mallik, R. C. Thermoelectric Properties of Co Substituted Synthetic Tetrahedrite. Acta Mater. 2015, 100, 266-274.

(29) Harish, S.; Sivaprahasam, D.; Battabyal, M.; Gopalan, R. Phase Stability and Thermoelectric Properties of Cu10.5Zn1.5Sb4S13 Tetrahedrite. J. Alloys Compd. 2016, 667, 323-328.

(30) James, D. J.; Lu, X.; Morelli, D. T.; Brock, S. L. Solvothermal Synthesis of Tetrahedrite: Speeding Up the Process of Thermoelectric Material Generation. ACS Appl. Mater. Interfaces 2015, 7 (42), 2362323632.

(31) Guin, S. N.; Banerjee, S.; Sanyal, D.; Pati, S. K.; Biswas, K. Origin of the Order-Disorder Transition and the Associated Anomalous Change of Thermopower in AgBiS2 Nanocrystals: A Combined Experimental and Theoretical Study. Inorg. Chem. 2016, 55, 6323-6331.

(32) Gharleghi, A.; Chu, Y.-H.; Lin, F.-H.; Yang, Z.-R.; Pai, Y.-H.; Liu, C.-J. Optimization and Analysis of Thermoelectric Properties of Unfilled Co1-x - y Nix Fey Sb3 Synthesized via a Rapid Hydrothermal Procedure. ACS Appl. Mater. Interfaces 2016, 8, 5205-5215.

(33) Ren, W.; Cheng, C.; Ren, Z.; Zhong, Y. The Effect of the Precursor Nanopowder Size on the Thermoelectric Properties of Nanostructured BiSbTe Bulk Materials. Phys. B 2010, 405, 49314936.

(34) An, C.; Jin, Y.; Tang, K.; Qian, Y. Selective Synthesis and Characterization of Famatinite Nanofibers and Tetrahedrite Nanoflakes. J. Mater. Chem. 2003, 13, 301-303.

(35) Yang, L.; Chen, Z. G.; Hong, M.; Han, G.; Zou, J. Enhanced Thermoelectric Performance of Nanostructured Bi2Te3 through Significant Phonon Scattering. ACS Appl. Mater. Interfaces 2015, 7, 23694-23699.

(36) Wang, W.; Poudel, B.; Yang, J.; Wang, D. Z.; Ren, Z. F. HighYield Synthesis of Single-Crystalline Antimony Telluride Hexagonal Nanoplates Using a Solvothermal Approach. J. Am. Chem. Soc. 2005, 127, 13792-13793.

(37) Tai, G.; Zhou, B.; Guo, W. Structural Characterization and Thermoelectric Transport Properties of Uniform Single-Crystalline Lead Telluride Nanowires. J. Phys. Chem. C 2008, 112, 11314-11318.

(38) Mi, J. L.; Zhao, X. B.; Zhu, T. J.; Tu, J. P.; Cao, G. S. Solvothermal Synthesis and Electrical Transport Properties of Skutterudite CoSb3. J. Alloys Compd. 2006, 417, 269-272.

(39) Ramasamy, K.; Sims, H.; Butler, W. H.; Gupta, A. Selective Nanocrystal Synthesis and Calculated Electronic Structure of All Four Phases of Copper-Antimony-Sulfide. Chem. Mater. 2014, 26, 28912899.

(40) Chen, K.; Zhou, J.; Chen, W.; Zhou, P.; He, F.; Liu, Y. SizeDependent Synthesis of Cu12Sb4S13 Nanocrystals with Bandgap Tunability. Part. Part. Syst. Charact. 2015, 32, 999-1005.

(41) Suehiro, S.; Horita, K.; Yuasa, M.; Tanaka, T.; Fujita, K.; Ishiwata, Y.; Shimanoe, K.; Kida, T. Synthesis of Copper-AntimonySulfide Nanocrystals for Solution-Processed Solar Cells. Inorg. Chem. 2015, 54, 7840-7845.

(42) Wu, Y.; Qiao, X.; Fan, X.; Zhang, X.; Cui, S.; Wan, J. Facile Synthesis of Monodisperse Cu3SbSe4 Nanoparticles and Thermoelectric Performance of Cu3SbSe4 Nanoparticle-Based Materials. J. Nanopart. Res. 2015, 17, 1-7.

(43) Arachchige, I. U.; Wu, J.; Dravid, V. P.; Kanatzidis, M. G. Nanocrystals of the Quaternary Thermoelectric Materials: AgPb $\mathrm{mSbTem}+2(\mathrm{~m}=1-18)$ : Phase-Segregated or Solid Solutions? Adv. Mater. 2008, 20, 3638-3642.

(44) Dirmyer, M. R.; Martin, J.; Nolas, G. S.; Sen, A.; Badding, J. V. Thermal and Electrical Conductivity of Size-Tuned Bismuth Telluride Nanoparticles. Small 2009, 5, 933-937.
(45) Scheele, M.; Oeschler, N.; Meier, K.; Kornowski, A.; Klinke, C.; Weller, H. Synthesis and Thermoelectric Characterization of Bi2Te 3 Nanoparticles. Adv. Funct. Mater. 2009, 19, 3476-3483.

(46) Zhao, Y.; Burda, C. Chemical Synthesis of Bi0.5Sb1.5Te3 Nanocrystals and Their Surface Oxidation Properties. ACS Appl. Mater. Interfaces 2009, 1, 1259-1263.

(47) Chen, D.; Zhao, Y.; Chen, Y.; Wang, B.; Wang, Y.; Zhou, J.; Liang, Z. Hot-Injection Synthesis of Cu-Doped $\mathrm{Cu} 2 \mathrm{ZnSnSe} 4$ Nanocrystals to Reach Thermoelectric zT of 0.70 at $450{ }^{\circ} \mathrm{C}$. ACS Appl. Mater. Interfaces 2015, 7, 24403-24408.

(48) Zhang, G.; Kirk, B.; Jauregui, L. a; Yang, H.; Xu, X.; Chen, Y. P.; $\mathrm{Wu}, \mathrm{Y}$. Rational Synthesis of Ultrathin N-Type Bi2Te3 Nanowires with Enhanced Thermoelectric Properties. Nano Lett. 2012, 12, 5660.

(49) Fang, H.; Feng, T.; Yang, H.; Ruan, X.; Wu, Y. Synthesis and Thermoelectric Properties of Compositional- Modulated Lead Telluride - Bismuth Telluride Nanowire Heterostructures. Nano Lett. 2013, 13, 2058-2063.

(50) Martin, J.; Nolas, G. S.; Zhang, W.; Chen, L. PbTe Nanocomposites Synthesized from PbTe Nanocrystals. Appl. Phys. Lett. 2007, 90, 222112.

(51) Urban, J. J.; Talapin, D. V.; Shevchenko, E. V.; Murray, C. B. Self-Assembly of PbTe Quantum Dots into Nanocrystal Superlattices and Glassy Films. J. Am. Chem. Soc. 2006, 128, 3248-3255.

(52) Kim, D. H.; Mitani, T. Thermoelectric Properties of FineGrained Bi2Te3 Alloys. J. Alloys Compd. 2005, 399, 14-19.

(53) Toprak, M.; Zhang, Y.; Muhammed, M. Chemical Alloying and Characterization of Nanocrystalline Bismuth Telluride. Mater. Lett. 2003, 57, 3976-3982.

(54) Khan, A.; Saleemi, M.; Johnsson, M.; Han, L.; Nong, N. V.; Muhammed, M.; Toprak, M. S. Fabrication, Spark Plasma Consolidation, and Thermoelectric Evaluation of Nanostructured CoSb3. J. Alloys Compd. 2014, 612, 293-300.

(55) Yasukawa, M.; Ioroi, A.; Ikeuchi, K.; Kono, T. Thermoelectric Properties of La-Doped $\mathrm{SrPbO} 3$ Ceramics Prepared by Coprecipitation Method. Mater. Lett. 2004, 58, 3536-3539.

(56) Sotelo, A.; Rasekh, S.; Torres, M. A.; Bosque, P.; Madre, M. A.; Diez, J. C. Effect of Synthesis Methods on the Ca3Co4O9 Thermoelectric Ceramic Performances. J. Solid State Chem. 2015, 221, 247-254.

(57) Zhou, B.; Zhao, Y.; Pu, L.; Zhu, J. Microwave-Assisted Synthesis of Nanocrystalline Bi2Te3. Mater. Chem. Phys. 2006, 96, 192-196.

(58) Birkel, C. S.; Zeier, W. G.; Douglas, J. E.; Lettiere, B. R.; Mills, C. E.; Seward, G.; Birkel, A.; Snedaker, M. L.; Zhang, Y.; Snyder, G. J.; Pollock, T. M.; Seshadri, R.; Stucky, G. D. Rapid Microwave Preparation of Thermoelectric TiNiSn and TiCoSb Half-Heusler Compounds. Chem. Mater. 2012, 24, 2558-2565.

(59) Kitchen, H. J.; Vallance, S. R.; Kennedy, J. L.; Tapia-Ruiz, N.; Carassiti, L.; Harrison, A.; Whittaker, A. G.; Drysdale, T. D.; Kingman, S. W.; Gregory, D. H. Modern Microwave Methods in Solid-State Inorganic Materials Chemistry: From Fundamentals to Manufacturing. Chem. Rev. 2014, 114, 1170-1206.

(60) Kerner, R.; Palchik, O.; Gedanken, A. Sonochemical and Microwave-Assisted Preparations of $\mathrm{PbTe}$ and $\mathrm{PbSe}$. A Comparative Study. Chem. Mater. 2001, 13, 1413-1419.

(61) Anderson, M. E.; Bharadwaya, S. S. N.; Schaak, R. E. Modified Polyol Synthesis of Bulk-Scale Nanostructured Bismuth Antimony Telluride. J. Mater. Chem. 2010, 20, 8362-8367.

(62) Datta, A.; Paul, J.; Kar, A.; Patra, A.; Sun, Z.; Chen, L.; Martin, J.; Nolas, G. S. Facile Chemical Synthesis of Nanocrystalline Thermoelectric Alloys Based on Bi-Sb-Te-Se. Cryst. Growth Des. 2010, 10, 3983-3989.

(63) Sk, R.; Shirolkar, M. M.; Dhara, B.; Kulkarni, S.; Deshpande, A. Enhancing the Thermopower and Tuning the Resistivity in Bi2Se3 with Fe-Doping. Chem. Phys. Lett. 2015, 638, 94-98.

(64) Kaspar, K.; Pelz, U.; Hillebrecht, H. Polyol Synthesis of NanoBi2Te3. J. Electron. Mater. 2014, 43, 1200-1206. 
(65) Yang, L.; Hng, H. H.; Ma, J.; Zhu, T. J.; Zhao, X. B. Effects of $\mathrm{Co:Sb}$ Molar Ratio on Synthesis and Properties of Undoped CoSb3 Prepared via a Polyol Method. J. Electron. Mater. 2010, 39, 1543-1548.

(66) Gharleghi, A.; Liu, C. Rapid Fabrication and Transport Properties of N-Type Co4-xNixSb12 via Modified Polyol Process Synthesis Combined with Evacuated-and-Encapsulated Sintering. J. Alloys Compd. 2014, 592, 277-282.

(67) Gharleghi, A.; Chang, H. Z.; Chen, Y. C.; Yang, Y. W.; Liu, C. J. Fabrication and Thermoelectric Power Factor of CoSb3 Prepared Using Modified Polyol Process and Evacuated- and-Encapsulated Sintering. J. Electron. Mater. 2013, 42, 1564-1567.

(68) Yang, L.; Hng, H. H.; Li, D.; Yan, Q. Y.; Ma, J.; Zhu, T. J.; Zhao, X. B.; Huang, H. Thermoelectric Properties of P-Type CoSb3 Nanocomposites with Dispersed CoSb3 Nanoparticles. J. Appl. Phys. 2009, 106, 013705.

(69) Soni, A.; Yanyuan, Z.; Ligen, Y.; Aik, M.; Dresselhaus, M.; Xiong, Q. Enhanced Thermoelectric Properties of Solution Grown Bi 2Te3-xSex Nanoplatelet Composites. Nano Lett. 2012, 12, 12031209.

(70) Holder, C. F.; Rugen, E. E.; Anderson, M. E. Comparative Growth Mechanism Study for Two Thermoelectric Compounds. Nanomater. Energy 2014, 3, 206-214.

(71) Lemoine, P.; Bourgès, C.; Barbier, T.; Nassif, V.; Cordier, S.; Guilmeau, E. High Temperature Neutron Powder Diffraction Study of the Cu12Sb4S13 and Cu4Sn7S16 Phases. J. Solid State Chem. 2017, $247,83-89$.

(72) Tanaka, H. I.; Suekuni, K.; Umeo, K.; Nagasaki, T.; Sato, H.; Kutluk, G.; Nishibori, E.; Kasai, H.; Takabatake, T. Metal-Semiconductor Transition Concomitant with a Structural Transformation. J. Phys. Soc. Jpn. 2016, 85, 1-6.

(73) Wang, J.; Gu, M.; Bao, Y.; Li, X.; Chen, L. Quick Fabrication and Thermoelectric Properties of Cu12Sb4S13 Tetrahedrite. J. Electron. Mater. 2016, 45, 2274-2277.

(74) Li, J. F.; Liu, W. S.; Zhao, L. D.; Zhou, M. High-Performance Nanostructured Thermoelectric Materials. NPG Asia Mater. 2010, 2, $152-158$. 